\title{
Anti-Saccharomyces cerevisiae Antibody in Pediatric Crohn's Disease Patients without Mucosal Healing Is a Useful Marker of Mucosal Damage
}

\author{
Mi Jin Kim¹, Eunsil Kim ${ }^{1}$, Ben Kang ${ }^{2}$, Yoon Lee ${ }^{3}$, Eun-Suk Kang ${ }^{4}$, and Yon Ho Choe ${ }^{1}$ \\ ${ }^{1}$ Department of Pediatrics, Samsung Medical Center, Sungkyunkwan University School of Medicine, Seoul, ${ }^{2}$ Department of Pediatrics, \\ School of Medicine, Kyungpook National University, Daegu, ${ }^{3}$ Department of Pediatrics, Korea University Anam Hospital, Korea \\ University College of Medicine, and ${ }^{4}$ Department of Laboratory Medicine and Genetics, Samsung Medical Center, Sungkyunkwan \\ University School of Medicine, Seoul, Korea
}

\section{Article Info}

Received July 8, 2020

Revised September 24, 2020

Accepted September 25, 2020

Published online December 31, 2020

\section{Corresponding Author}

Yon Ho Choe

ORCID https://orcid.org/0000-0003-1525-7688

E-mail 1101016@skku.edu
Background/Aims: We evaluated whether anti-Saccharomyces cerevisiae antibody (ASCA) titers are associated with diagnostic findings, disease activity, Paris classification phenotypes, and persistence after infliximab (IFX) treatment in children with Crohn's disease (CD). We also investigated the role of ASCA as a predictor of mucosal healing $(\mathrm{MH})$ and clinical remission $(\mathrm{CR})$. Methods: This study included $61 \mathrm{CD}$ patients aged 19 years or younger who were diagnosed and treated between September 2010 and January 2019 and followed for at least 1 year. ASCA was regularly measured at the diagnosis of $C D$ and at least 1 year after IFX therapy.

Results: The average follow-up period was $3.8 \pm 3.4$ years (range, 1.0 to 7.2 years). Regression analysis showed that the ASCA titer was the only factor associated with Simple Endoscopic Score for Crohn's Disease (SES-CD) or CR among all the parameters. In patients who had achieved $\mathrm{MH}(\mathrm{SES}-\mathrm{CD}=0)$, ASCA immunoglobulin $\mathrm{G}(\mathrm{lgG})$ was not associated with $\mathrm{MH}$, but in patients without MH, ASCA IgG was associated with SES-CD $(p=0.005)$ and $C R(p<0.001)$. The cutoff value of ASCA IgG in patients with CR was 21.8 units. However, there was no difference in the relapse rate between the ASCA IgG-positive and -negative groups during the follow-up period.

Conclusions: In patients who have not achieved MH, ASCA IgG is closely related to mucosal damage and CR. Unlike Western studies, ASCA IgG may be more helpful in predicting prognosis than immunoglobulin A in Korean patients, but it is not an appropriate indicator to predict the relapse of CD. (Gut Liver 2021;15:763-770)

Key Words: Crohn disease; Anti-Saccharomyces cerevisiae; Children; Marker; Mucosal healing

\section{INTRODUCTION}

The pathogenesis of Crohn's disease (CD) is not clearly defined, but the prevalence of pediatric $\mathrm{CD}$ worldwide, including Korea, is on the rise. ${ }^{1-4}$ Despite the availability of many serum biomarkers, there is a lack of reliable tools to predict CD prognosis. ${ }^{5,6}$ In 1988, Main et al. ${ }^{7}$ first reported elevated anti-Saccharomyces cerevisiae antibody (ASCA) levels in CD. ASCA is a useful tool to distinguish CD from ulcerative colitis, but its value as a prognostic marker is emerging gradually. ${ }^{8-10}$

ASCA is generally common in younger age groups, those with ileal involvement, fibrostenosis, and more aggressive and complex disease behavior. ${ }^{11-13}$ Several studies have investigated whether ASCA titers are altered with disease activity, and successful treatment has shown that its titers are stable or decreased. ${ }^{14-16}$ However, few studies have investigated the association between ASCA titers and disease prognosis, relapse and biological treatment in children with $\mathrm{CD}^{17-19}$ The introduction of various biological agents, including monoclonal antibodies that block the inflammatory cytokine tumor necrosis factor- $\alpha$, has altered the treatment and management options for CD. Serological markers can be used to assess the therapeutic response 
of these biological agents in pediatric cases of CD. ${ }^{20,21}$

Our study evaluated whether ASCA titers are associated with diagnostic value, disease activity, Paris classification phenotypes, durability over time after infliximab (IFX) treatment in children with $\mathrm{CD}$. We also investigated the role of ASCA as a predictor of mucosal healing $(\mathrm{MH})$ and clinical remission (CR).

\section{MATERIALS AND METHODS}

\section{Patients and data collection}

A comprehensive medical chart review was performed for all children with CD who were aged below 19 years at the Samsung Medical Center and had quantitative ASCA immunoglobulin A (IgA) and immunoglobulin G (IgG) titers at diagnosis and follow-up. This study was conducted in patients who were treated with IFX for at least 1 year between September 2010 and January 2019.

Paris classification was used to determine the disease phenotype. ${ }^{22}$ The pediatric Crohn's disease activity index (PCDAI), serum albumin, hematocrit, erythrocyte sedimentation rate and $\mathrm{C}$-reactive protein values were determined concurrently with the ASCA titers. The PCDAI score ranges from zero to 100 , with a score $>10$ indicating active disease. $^{23}$ Simple Endoscopic Score for Crohn's Disease (SES-CD) was also measured by colonoscopy at diagnosis and at least 1 year after IFX treatment, followed by 1 to 2 years intervals. Patients in CR were identified based on PCDAI scores less than 10. Patients with $\mathrm{MH}$ were identified based on SES-CD less than 0. Patients were also required to have data on serum IFX trough levels, obtained from blood samples taken immediately before the IFX infusion.

The data were collected from all the eligible patients including those with ASCA and simultaneous SES-CD. Approval was obtained from the Institutional Review Board of the Samsung Medical Center Committee on Clinical Investigation (IRB number: 2019-12-152). The requirement for obtaining informed consent from the patients was waived due to the retrospective nature of the study.

\section{ASCA IgA and IgG measurements}

ASCA IgA and IgG were quantified using commercially available standard calibrated enzyme-linked immunosorbent assay kits, and the tests were performed according to the manufacturer's protocol (ASCA IgG and ASCA IgA; QUANTA Lite ${ }^{\mathrm{TM}}$, INOVA Diagnostics, San Diego, CA, USA). Results of ASCA IgA and IgG testing were classified as negative ( 0.0 to 20.0 units), equivocal (20.1 to 24.9 units), or positive ( $\geq 25$ units).

\section{Primary outcome}

The primary outcome of this study was the role of ASCA according to disease severity before and after IFX treatment, such as Paris classification phenotypes, laboratory findings, and SES-CD, especially in pediatric CD patients. ASCA titers at diagnosis were compared with those after at least 1 year of IFX treatment, and their correlation with $\mathrm{CR}$ and endoscopic $\mathrm{MH}$ were evaluated.

\section{Statistical analysis}

Data were expressed as the mean and standard deviation (continuous variables) and as frequencies and percentages (categorical variables). The chi-square test and Mann-Whitney test were used to compare categorical and quantitative variables. When comparing measurements at baseline and after IFX treatment, the Wilcoxon signedrank test was used. A receiver operating characteristic analysis was performed to calculate the cutoff value of ASCA IgG for CR. Kaplan-Meier analysis was used to estimate the increase in free survival rate during long-term follow-up.

Simple and multiple linear regression analyses were used to identify the factors associated with $\mathrm{MH}$ and CR. A multivariate linear regression analysis was then conducted using a stepwise selection procedure with a $5 \%$ significance level for a covariate to enter or remain in the model. Linear correlation was estimated using the Pearson correlation and represented as correlation coefficients ( $r$ ) on a scatter plot. The results were also expressed as probabilities. All

Table 1. Clinical Characteristics of Patients with Crohn's Disease

\begin{tabular}{|c|c|}
\hline Characteristics & Value $(n=61)$ \\
\hline Male sex & $41(67.2)$ \\
\hline Age at diagnosis, yr & $14.6 \pm 2.3$ \\
\hline \multicolumn{2}{|l|}{ Paris location } \\
\hline L1 (distal 1/3 of ileum \pm caecum) & $3(4.9)$ \\
\hline L2 (colon) & 2 (3.3) \\
\hline L3 (ileocolonic) & 56 (91.8) \\
\hline L4 (upper GI tract involvement) & $29(47.5)$ \\
\hline \multicolumn{2}{|l|}{ Paris behavior } \\
\hline B1 (non-stricturing, non-penetrating) & 48 (78.7) \\
\hline $\mathrm{B} 2$ (stricturing) & $12(19.7)$ \\
\hline B3 (penetrating) & $1(1.6)$ \\
\hline Perianal disease & 32 (52.5) \\
\hline Dose intensification during IFX treatment & 7 (11.5) \\
\hline Concomitant AZA treatment & 55 (90.2) \\
\hline Corticosteroid usage & $3(4.9)$ \\
\hline Any lifelong CD-related GI surgery & 2 (3.3) \\
\hline Growth impairment & $6(9.8)$ \\
\hline Family history of IBD & 5 (8.2) \\
\hline
\end{tabular}

Data are presented as number $(\%)$ or mean \pm SD.

GI, gastrointestinal; IFX, infliximab; AZA, azathioprine; CD, Crohn's disease; IBD, inflammatory bowel disease. 
tests were two-sided, and p-values $<0.05$ are considered statistically significant. All analyses were performed using SPSS, statistical software SPSS version 25 (IBM Corp., Armonk, NY, USA).

\section{RESULTS}

\section{Baseline characteristics}

Serial 167 measurements of ASCA IgA and IgG titers

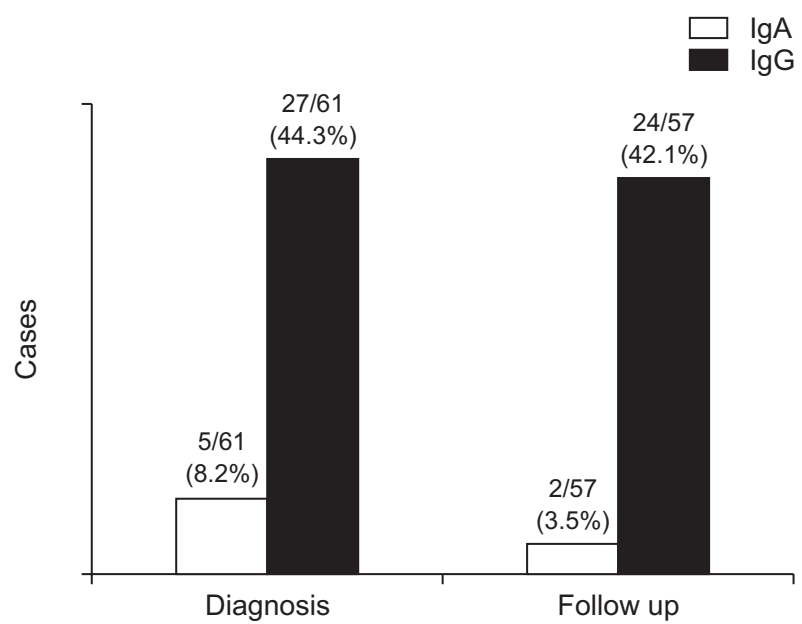

Fig. 1. ASCA-positive cases at diagnosis and follow-up. ASCA IgApositive results were found in five cases, and ASCA IgG-positive results were found in 27 cases at diagnosis. During follow-up, two ASCA IgA-positive and 24 ASCA IgG-positive patients were detected. ASCA, anti-Saccharomyces cerevisiae antibody; IgA, immunoglobulin $A ; \lg G$, immunoglobulin $G$. were documented in 61 children with $\mathrm{CD}$. The mean age at diagnosis was $14.6 \pm 2.3$ years, with age ranging from 8.8 to 18.9 years, and included 41 boys. The patients' characteristics are summarized in Table 1. Disease phenotype was determined according to the Paris classification.

The mean duration between initial diagnosis and the follow-up was $3.8 \pm 3.4$ years. There were 32 out of 61 (52.5\%) ASCA-positive (either IgA or IgG) CD patients at diagnosis and 26 out of $57(45.6 \%)$ patients at follow-up after IFX treatment. Five (8.2\%) ASCA IgA-positive and 27 (44.3\%) ASCA IgG-positive CD patients were documented at diagnosis. At the time of follow-up, two (3.5\%) ASCA

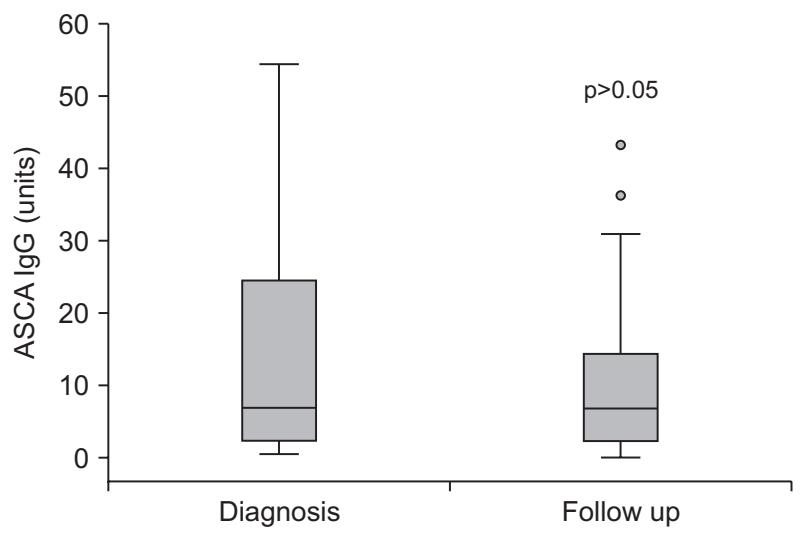

Fig. 2. Durability of the ASCA titer over time. The ASCA titer at diagnosis was $14.3 \pm 14.4$ units and the titer at follow-up was $10.5 \pm 10.0$ units. The mean duration of follow-up was $3.8 \pm 3.4$ and ranged from 1.0 to 7.2 years. There was no significant difference in the titer of ASCA before and after infliximab treatment.

ASCA, anti-Saccharomyces cerevisiae antibody; IgG, immunoglobulin G.

Table 2. Simple and Multiple Linear Regression Analyses of Factors Associated with SES-CD

\begin{tabular}{|c|c|c|c|c|c|c|c|c|}
\hline \multirow{2}{*}{ Variable } & \multicolumn{4}{|c|}{ Simple linear regression analysis } & \multicolumn{4}{|c|}{ Multiple linear regression analysis } \\
\hline & $B \pm S E$ & $95 \% \mathrm{Cl}$ & Partial $\mathrm{R}^{2}$ & p-value & $B \pm S E$ & $95 \% \mathrm{Cl}$ & Partial $R^{2}$ & $\mathrm{p}$-value \\
\hline Sex (male vs female) & $-3.500 \pm 2.224$ & -8.080 to 1.080 & 0.090 & 0.128 & & & & \\
\hline Age at diagnosis, yr & $0.107 \pm 0.411$ & -0.739 to 0.953 & 0.003 & 0.797 & & & & \\
\hline Upper GI tract involvement (yes vs no) & $0.767 \pm 2.050$ & -3.456 to 4.989 & 0.006 & 0.712 & & & & \\
\hline B1 disease behavior (yes vs no) & $-1.645 \pm 2.213$ & -6.203 to 2.913 & 0.022 & 0.464 & & & & \\
\hline Perianal modifiers (yes vs no) & $2.267 \pm 2.005$ & -1.864 to 6.397 & 0.049 & 0.269 & & & & \\
\hline Linear growth failure (yes vs no) & $-0.989 \pm 2.869$ & -6.898 to 4.920 & 0.005 & 0.733 & & & & \\
\hline $\mathrm{WBC}, / \mu \mathrm{L}$ & $0.000 \pm 0.000$ & -0.001 to 0.000 & 0.022 & 0.463 & & & & \\
\hline Hct, \% & $0.006 \pm 0.017$ & -0.030 to 0.041 & 0.004 & 0.744 & & & & \\
\hline Platelet count, $/ \mu \mathrm{L}$ & $0.001 \pm 0.010$ & -0.020 to 0.022 & 0.000 & 0.920 & & & & \\
\hline Albumin, g/dL & $-1.601 \pm 1.789$ & -5.286 to 2.084 & 0.031 & 0.379 & & & & \\
\hline $\mathrm{ESR}, \mathrm{mm} / \mathrm{hr}$ & $0.004 \pm 0.033$ & -0.063 to 0.071 & 0.001 & 0.899 & & & & \\
\hline $\mathrm{CRP}, \mathrm{mg} / \mathrm{dL}$ & $0.089 \pm 0.295$ & -0.519 to 0.697 & 0.004 & 0.766 & & & & \\
\hline IFX trough level, $\mu \mathrm{g} / \mathrm{mL}$ & $0.130 \pm 0.205$ & -0.294 to 0.553 & 0.017 & 0.533 & & & & \\
\hline ASCA IgA, units & $0.310 \pm 0.310$ & 0.084 to 0.535 & 0.243 & $0.009^{*}$ & & & & \\
\hline ASCA IgG, units & $0.196 \pm 0.064$ & 0.064 to 0.328 & 0.273 & $0.005^{*}$ & $0.219 \pm 0.057$ & 0.103 to 0.336 & 0.465 & $0.001 *$ \\
\hline
\end{tabular}

SES-CD, Simple Endoscopic Score for Crohn's Disease; SE, standard error; Cl, confidence interval; GI, gastrointestinal; WBC, white blood cell; Hct, hematocrit; ESR, erythrocyte sedimentation rate; CRP, C-reactive protein; IFX, infliximab; ASCA, anti-Saccharomyces cerevisiae antibody; IgA, immunoglobulin $A$; lgG, immunoglobulin $G$.

$* p<0.05$. 
IgA-positive and 24 (42.1\%) ASCA IgG-positive patients were identified (Fig. 1).

\section{Durability of ASCA titer}

There was no statistically significant difference in the titer of ASCA before and after IFX treatment. The mean duration of follow-up was 3.8 \pm 3.4 years, ranging from 1.0 to 7.2 years. The ASCA titer at diagnosis was $14.3 \pm 14.4$ units and the titer at follow-up was $10.5 \pm 10.0$ units $(\mathrm{p}>0.05)$ (Fig. 2).

\section{Factors associated with $\mathrm{MH}$ and $\mathrm{CR}$}

The linear regression analysis was used to determine factors associated with $\mathrm{MH}$ and $\mathrm{CR}$ in $61 \mathrm{CD}$ patients. Factors included sex, age at diagnosis, Paris classification phenotypes, linear growth failure, various laboratory tests, IFX trough levels and ASCA IgA and IgG. In simple and multiple linear regression analyses, ASCA IgA and IgG was the only factor associated with SES-CD (Table 2). Analyses of factors associated with PCDAI also showed similar results (Table 3).

Table 3. Simple and Multiple Linear Regression Analyses of Factors Associated with PCDAl

\begin{tabular}{|c|c|c|c|c|c|c|c|c|}
\hline \multirow{2}{*}{ Variable } & \multicolumn{4}{|c|}{ Simple linear regression analysis } & \multicolumn{4}{|c|}{ Multiple linear regression analysis } \\
\hline & $B \pm S E$ & $95 \% \mathrm{Cl}$ & Partial $R^{2}$ & $\mathrm{p}$-value & $\mathrm{B} \pm \mathrm{SE}$ & $95 \% \mathrm{Cl}$ & Partial $\mathrm{R}^{2}$ & $\mathrm{p}$-value \\
\hline Sex (male vs female) & $0.268 \pm 3.639$ & -7.226 to 7.762 & 0.000 & 0.942 & & & & \\
\hline Age at diagnosis, yr & $-0.051 \pm 0.642$ & -1.373 to 1.271 & 0.000 & 0.938 & & & & \\
\hline Upper GI tract involvement (yes vs nol & $2.375 \pm 3.174$ & -4.162 to 8.912 & 0.022 & 0.461 & & & & \\
\hline B1 disease behavior (yes vs no) & $-1.234 \pm 3.484$ & -8.408 to 5.941 & 0.005 & 0.726 & & & & \\
\hline Perianal modifiers (yes vs no) & $5.750 \pm 2.996$ & -0.421 to 11.921 & 0.128 & 0.066 & & & & \\
\hline Linear growth failure (yes vs no) & $-3.016 \pm 4.448$ & -12.178 to 6.145 & 0.018 & 0.504 & & & & \\
\hline $\mathrm{WBC}, / \mu \mathrm{L}$ & $-0.001 \pm 0.001$ & -0.002 to 0.000 & 0.055 & 0.240 & & & & \\
\hline Hct, \% & $-0.014 \pm 0.027$ & -0.069 to 0.042 & 0.010 & 0.617 & & & & \\
\hline Platelet count, / $/ \mathrm{L}$ & $-0.020 \pm 0.015$ & -0.051 to 0.012 & 0.061 & 0.213 & & & & \\
\hline Albumin, g/dL & $-4.712 \pm 2.676$ & -10.224 to 0.799 & 0.110 & 0.090 & & & & \\
\hline $\mathrm{ESR}, \mathrm{mm} / \mathrm{hr}$ & $0.001 \pm 0.051$ & -0.104 to 0.106 & 0.000 & 0.985 & & & & \\
\hline $\mathrm{CRP}, \mathrm{mg} / \mathrm{dL}$ & $0.212 \pm 0.460$ & -0.735 to 1.159 & 0.008 & 0.649 & & & & \\
\hline IFX trough level, $\mu \mathrm{g} / \mathrm{mL}$ & $-0.113 \pm 0.328$ & -0.791 to 0.565 & 0.005 & 0.734 & & & & \\
\hline ASCA IgA, units & $0.492 \pm 0.170$ & 0.142 to 0.842 & 0.252 & $0.008^{*}$ & & & & \\
\hline ASCA IgG, units & $0.377 \pm 0.090$ & 0.192 to 0.562 & 0.414 & $<0.001 *$ & $0.377 \pm 0.090$ & 0.192 to 0.562 & 0.414 & $<0.001 *$ \\
\hline
\end{tabular}

PCDAl, pediatric Crohn's disease activity index; SE, standard error; $\mathrm{Cl}$, confidence interval; GI, gastrointestinal; WBC, white blood cell; Hct, hematocrit; ESR, erythrocyte sedimentation rate; CRP, C-reactive protein; IFX, infliximab; ASCA, anti-Saccharomyces cerevisiae antibody; IgA, immunoglobulin A; IgG, immunoglobulin $G$.

${ }^{*} \mathrm{p}<0.05$.
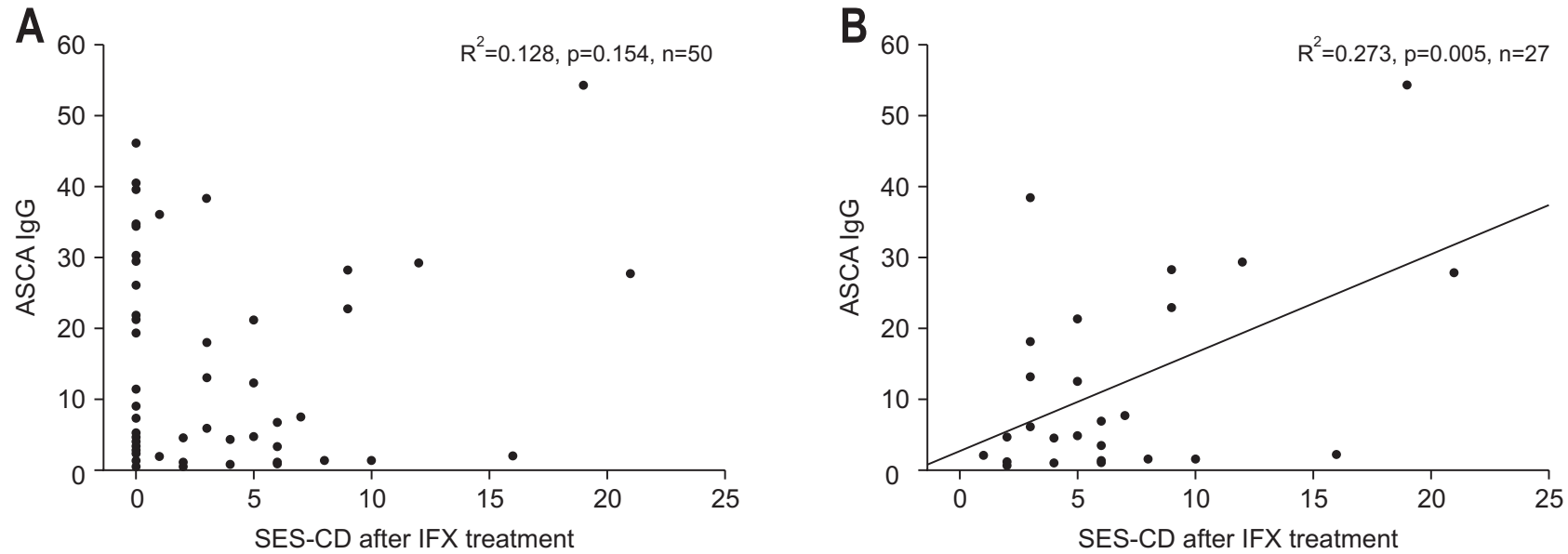

Fig. 3. Correlation between the ASCA titer and SES-CD after IFX treatment. There was no statistically significant difference in the correlation between $\mathrm{MH}$ based on SES-CD and the ASCA titer after IFX treatment (A). A significant correlation was observed after eliminating ASCA titers in patients with an SES-CD score of 0 (B).

ASCA, anti-Saccharomyces cerevisiae antibody; SES-CD, Simple Endoscopic Score for Crohn Disease; IFX, infliximab; MH, mucosal healing; IgG, immunoglobulin $\mathrm{G}$. 


\section{Correlation between ASCA titer and SES-CD score or CR after IFX treatment}

There was no statistically significant difference in correlation between $\mathrm{MH}$ based on SES-CD and ASCA titer after IFX treatment (Fig. 3A). However, when ASCA titers of patients with SES-CD score of 0 were removed, a significant correlation was found between the two parameters ( $\mathrm{p}=0.005)$ (Fig. 3B). The correlation between $\mathrm{CR}$ through PCDAI and ASCA IgG after IFX treatment was also observed between the two indicators ( $\mathrm{p}<0.001)$ (Fig. 4). There was no statistically significant difference in the rate of bowel surgery depending on whether ASCA IgG was positive or not.

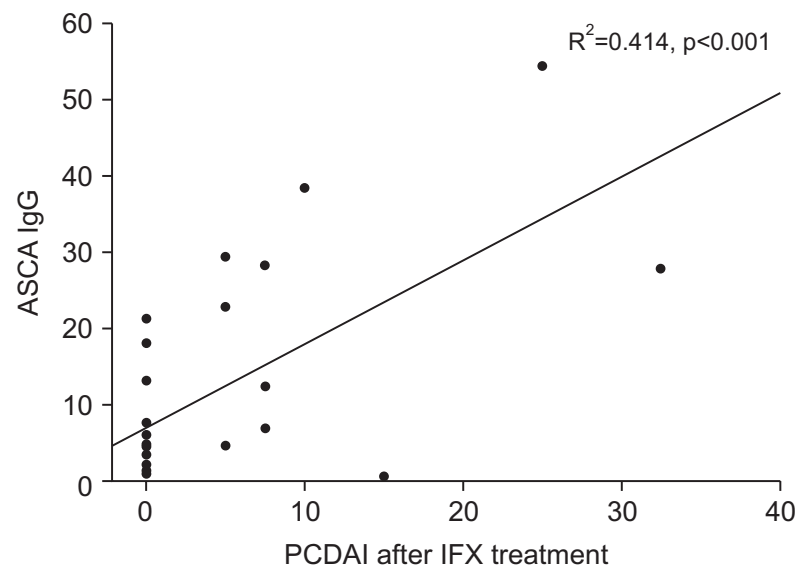

Fig. 4. Correlation between the ASCA titer and clinical remission (CR) after infliximab (IFX) treatment. The correlation between CR through PCDAl and the ASCA titer after IFX treatment was observed between the two indicators.

ASCA, anti-Saccharomyces cerevisiae antibody; PCDAl, pediatric Crohn's disease activity index; IgG, immunoglobulin $G$.

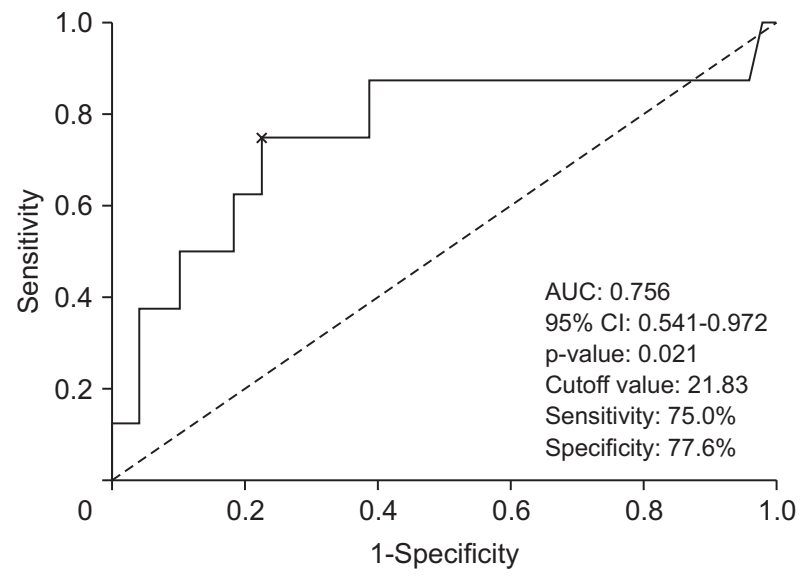

Fig. 5. Receiver operating characteristic curve for estimating the diagnostic accuracy of the ASCA titer for the presumptive clinical remission of Crohn's disease. The cutoff value of ASCA IgG for clinical remission was 21.8 units.

ASCA, anti-Saccharomyces cerevisiae antibody; IgG, immunoglobulin $\mathrm{G}$; AUC, area under the curve; $\mathrm{Cl}$, confidence interval.

\section{Receiver operating curve for estimation of the diagnostic accuracy of ASCA titer for presumptive CR}

The area under the receiver operating characteristic curve for ASCA IgG was associated with CR of CD patients: 0.756 (95\% confidence interval, 0.541 to 0.972 ) (Fig. 5). The cutoff of ASCA IgG associated with CR with the maximum sensitivity and specificity was 21.8 units (sensitivity, 75.0\%; specificity, 77.6\%).

\section{Survival plots for disease relapse during follow-up}

After a median follow-up of 3.8 years (range, 1 to 7.2 years), $57.4 \%$ (35/61) of patients who were ASCA IgG positive had experienced clinical relapse. According to the Kaplan-Meier survival analysis of ASCA IgG-positive patients, the estimated cumulative relapse rates at 1, 2, 4, and 6 years were $17.3 \%, 31.2 \%, 58.8 \%$, and $71.3 \%$, respectively. Among ASCA IgG-negative patients, 45.9\% (28/61) had experienced clinical relapse. According to the KaplanMeier survival analysis of ASCA IgG-negative patients, the cumulative relapse rates at 1, 2, 4, and 6 years were $16.5 \%$, $29.9 \%, 27.6 \%$, and $48.5 \%$, respectively. No statistically significant differences were found between ASCA IgG positivity and recurrence rate during the follow-up period (Fig. 6).

\section{DISCUSSION}

Several studies have confirmed the association between ASCA antibodies and CD. ASCA antibodies have been known to facilitate the diagnosis and prediction of disease

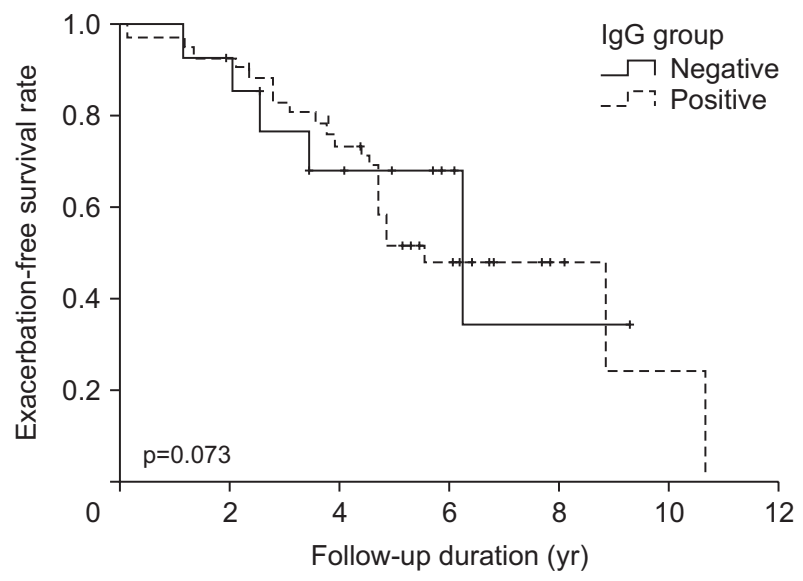

Fig. 6. Survival plots showing disease relapse during follow-up. There was no statistically significant difference between ASCA positivity and the recurrence rate during the follow-up period.

ASCA, anti-Saccharomyces cerevisiae antibody; IgG, immunoglobulin G. 
progression in CD patients. ${ }^{12,14,15}$ However, the association between ASCA titer and MH based on endoscopy is unclear. This study was conducted in patients who were treated with IFX for at least 1 year. The potential of IFX, not only to induce but also to maintain remission, has been well known in adult and pediatric CD patients. It is no longer unfamiliar to set the therapeutic goal of $\mathrm{CD}$ based on $\mathrm{MH}$ as well as CR. In addition to endoscopy used to identify $\mathrm{MH}$, the development of noninvasive and reliable tools is an ongoing challenge. This study is the first to explore the utility of ASCA as a noninvasive tool in $\mathrm{MH}$ screening. Although the association between ASCA IgG and $\mathrm{MH}$ was not completely correlated in this study, it was shown that there was an association between ASCA IgG and SES-CD, which was a factor predicting MH. Further, it is not clear whether serum ASCA titers change according to $\mathrm{CD}$ activity following treatment and long-term followup. Our study showed that the measurement of ASCA titer facilitates the assessment of $\mathrm{CR}$ and $\mathrm{MH}$ in CD patients. We also demonstrated the persistence of ASCA after treatment.

Ruemmele et al. ${ }^{14}$ and Canani et al. ${ }^{24}$ suggest that CD behavior is not associated with ASCA titers in pediatric patients. ${ }^{14,24}$ However, several studies have reported high ASCA titers among more severe CD patients. ${ }^{25,26}$ In our study, ASCA is closely related to disease activity and mucosal damage status. Pediatric $\mathrm{CD}$ patients who carried high ASCA IgG titers manifested a more clinically severe disease and a higher risk of developing extensive endoscopic luminal disease. Therefore, careful monitoring is required when the titer is high during initial ASCA measurements. The one difference from Western studies is that ASCA $\operatorname{IgG}$, rather than IgA, is a useful predictor of complicated disease behavior in Korea. ${ }^{11,19}$

Previous studies have suggested a link between ASCA titers and Paris classification phenotypes, that is, positive ASCA titers may be associated with ileocecal disease or correlate with both L3 and L4 ${ }^{19,27}$ However, we found no significant association between Paris classification phenotypes and positive ASCA status. Considering that most of the previous studies investigating ASCA have focused on Westerners, our results may be attributed to ethnic differences.

Rieder et $a .^{28}$ found that ASCA titer decreased after corticosteroid therapy. However, Teml et al. ${ }^{15}$ analyzed ASCA levels in CD patients before and after treatment with either steroids or mesalamine, and reported that the ASCA levels did not change over a period of 2 to 9 months. Other investigators also showed that several antibodies in CD remained stable after 4 months of IFX therapy. ${ }^{29}$ Our data showed that ASCA titers remained stable even during the follow-up period up to 7 years and it was more reliable than those of other short-term studies. Repeated measurements of ASCA titers after diagnosis are unlikely to be useful.

Chandrakumar et al. ${ }^{19}$ showed that CD patients who were positive for ASCA IgA and IgG did not show a greater relapse rate than patients with negative titers. These results are similar to our study. There was no significant difference in relapse rate between ASCA IgG-positive and -negative patients, suggesting that ASCA is not an appropriate indicator to predict $\mathrm{CD}$ relapse.

The study is limited by a relatively small sample size and retrospective study design. Fecal calprotectin is a useful noninvasive marker of $\mathrm{MH}$ and has already been used to monitor therapeutic success in many studies. Unfortunately, in this study, fecal calprotectin was not used as a variable because it was not available during some periods. However, it is significant that ASCA has been proposed as a marker of $\mathrm{MH}$ after IFX treatment of children with CD.

In conclusion, the results of this study reveal the importance of ASCA as a noninvasive tool for monitoring disease activity and mucosal damage status in Korean children with $\mathrm{CD}$. In patients who have not achieved $\mathrm{MH}$, ASCA IgG is closely related to mucosal damage status and CR. Therefore, careful monitoring is required when the titer is high during initial ASCA measurements. Unlike Western studies, ASCA IgG may be more helpful in predicting prognosis than IgA in Korean patients, but it is not an appropriate indicator to predict the relapse of CD. Further prospective studies involving a large number of patients are needed to better define the value of ASCA in children with CD.

\section{CONFLICTS OF INTEREST}

No potential conflict of interest relevant to this article was reported.

\section{ACKNOWLEDGEMENTS}

This work was supported by the National Research Foundation of Korea (NRF) grant funded by the Korea government (MSIT) (No. 2020R1A2C2007192).

\section{AUTHOR CONTRIBUTIONS}

Study concept and design: M.J.K. Data acquisition: Y.L. Data analysis and interpretation: B.K. Drafting of the man- 
uscript; critical revision of the manuscript for important intellectual content: E.S.K. Statistical analysis: E.K. Administrative, technical, or material support; study supervision: Y.H.C. Approval of final manuscript: all authors.

\section{ORCID}

Mi Jin Kim https://orcid.org/0000-0002-4505-4083

Eunsil Kim https://orcid.org/0000-0003-2012-9867

Ben Kang https://orcid.org/0000-0002-8516-9803

Yoon Lee https://orcid.org/0000-0001-9521-3575

Eun-Suk Kang https://orcid.org/0000-0001-6386-6520

Yon Ho Choe https://orcid.org/0000-0003-1525-7688

\section{REFERENCES}

1. Hong SJ, Cho SM, Choe BH, et al. Characteristics and incidence trends for pediatric inflammatory bowel disease in Daegu-Kyungpook province in Korea: a multi-center study. J Korean Med Sci 2018;33:e132.

2. Benchimol EI, Bernstein CN, Bitton A, et al. Trends in epidemiology of pediatric inflammatory bowel disease in Canada: distributed network analysis of multiple population-based provincial health administrative databases. Am J Gastroenterol 2017;112:1120-1134.

3. El-Matary W, Moroz SP, Bernstein CN. Inflammatory bowel disease in children of Manitoba: 30 years' experience of a tertiary center. J Pediatr Gastroenterol Nutr 2014;59:763766.

4. Lee KM, Lee JM. Crohn's disease in Korea: past, present, and future. Korean J Intern Med 2014;29:558-570.

5. Zhou G, Song Y, Yang W, et al. ASCA, ANCA, ALCA and many more: are they useful in the diagnosis of inflammatory bowel disease? Dig Dis 2016;34:90-97.

6. Levine A. Pediatric inflammatory bowel disease: is it different? Dig Dis 2009;27:212-214.

7. Main J, McKenzie H, Yeaman GR, et al. Antibody to Saccharomyces cerevisiae (bakers' yeast) in Crohn's disease. BMJ 1988;297:1105-1106.

8. Lewis JD. The utility of biomarkers in the diagnosis and therapy of inflammatory bowel disease. Gastroenterology 2011;140:1817-1826.

9. El-Matary W, Dupuis K, Sokoro A. Anti-Saccharomyces cerevisiae antibody titres correlate well with disease activity in children with Crohn's disease. Acta Paediatr 2015;104:827830.

10. Kim BC, Park S, Han J, Kim JH, Kim TI, Kim WH. Clinical significance of anti-Saccharomyces cerevisiae antibody (ASCA) in Korean patients with Crohn's disease and its relationship to the disease clinical course. Dig Liver Dis 2007;39:610-616.

11. Peeters M, Joossens S, Vermeire S, Vlietinck R, Bossuyt X, Rutgeerts P. Diagnostic value of anti-Saccharomyces cerevisiae and antineutrophil cytoplasmic autoantibodies in inflammatory bowel disease. Am J Gastroenterol 2001;96:730734.

12. Forcione DG, Rosen MJ, Kisiel JB, Sands BE. Anti-Saccharomyces cerevisiae antibody (ASCA) positivity is associated with increased risk for early surgery in Crohn's disease. Gut 2004;53:1117-1122.

13. Zhang Z, Li C, Zhao X, et al. Anti-Saccharomyces cerevisiae antibodies associate with phenotypes and higher risk for surgery in Crohn's disease: a meta-analysis. Dig Dis Sci 2012;57:2944-2954.

14. Ruemmele FM, Targan SR, Levy G, Dubinsky M, Braun J, Seidman EG. Diagnostic accuracy of serological assays in pediatric inflammatory bowel disease. Gastroenterology 1998;115:822-829.

15. Teml A, Kratzer V, Schneider B, et al. Anti-Saccharomyces cerevisiae antibodies: a stable marker for Crohn's disease during steroid and 5-aminosalicylic acid treatment. Am J Gastroenterol 2003;98:2226-2231.

16. Oshitani N, Hato F, Matsumoto T, et al. Decreased antiSaccharomyces cerevisiae antibody titer by mesalazine in patients with Crohn's disease. J Gastroenterol Hepatol 2000;15:1400-1403.

17. Esters N, Vermeire S, Joossens S, et al. Serological markers for prediction of response to anti-tumor necrosis factor treatment in Crohn's disease. Am J Gastroenterol 2002;97:14581462.

18. Dubinsky MC, Mei L, Friedman M, et al. Genome wide association (GWA) predictors of anti-TNFalpha therapeutic responsiveness in pediatric inflammatory bowel disease. Inflamm Bowel Dis 2010;16:1357-1366.

19. Chandrakumar A, Georgy M, Agarwal P, 't Jong GW, ElMatary W. Anti-Saccharomyces cerevisiae antibodies as a prognostic biomarker in children with Crohn disease. J Pediatr Gastroenterol Nutr 2019;69:82-87.

20. Miheller P, Kiss LS, Juhasz M, Mandel M, Lakatos PL. Recommendations for identifying Crohn's disease patients with poor prognosis. Expert Rev Clin Immunol 2013;9:65-75.

21. Rieder F, Hahn P, Finsterhoelzl L, et al. Clinical utility of anti-glycan antibodies in pediatric Crohn's disease in comparison with an adult cohort. Inflamm Bowel Dis 2012;18:12211231.

22. Levine A, Griffiths A, Markowitz J, et al. Pediatric modification of the Montreal classification for inflammatory bowel disease: the Paris classification. Inflamm Bowel Dis 2011;17:1314-1321.

23. Hyams J, Markowitz J, Otley A, et al. Evaluation of the pedi- 
atric Crohn disease activity index: a prospective multicenter experience. J Pediatr Gastroenterol Nutr 2005;41:416-421.

24. Canani RB, Romano MT, Greco L, et al. Effects of disease activity on anti-Saccharomyces cerevisiae antibodies: implications for diagnosis and follow-up of children with Crohn's disease. Inflamm Bowel Dis 2004;10:234-239.

25. Vasiliauskas EA, Kam LY, Karp LC, Gaiennie J, Yang H, Targan SR. Marker antibody expression stratifies Crohn's disease into immunologically homogeneous subgroups with distinct clinical characteristics. Gut 2000;47:487-496.

26. Halfvarson J, Standaert-Vitse A, Jarnerot G, et al. AntiSaccharomyces cerevisiae antibodies in twins with inflam- matory bowel disease. Gut 2005;54:1237-1243.

27. Quinton JF, Sendid B, Reumaux D, et al. Anti-Saccharomyces cerevisiae mannan antibodies combined with antineutrophil cytoplasmic autoantibodies in inflammatory bowel disease: prevalence and diagnostic role. Gut 1998;42:788-791.

28. Rieder F, Lopez R, Franke A, et al. Characterization of changes in serum anti-glycan antibodies in Crohn's disease: a longitudinal analysis. PLoS One 2011;6:e18172.

29. Targan SR, Landers CJ, Yang H, et al. Antibodies to CBir1 flagellin define a unique response that is associated independently with complicated Crohn's disease. Gastroenterology 2005;128:2020-2028. 